\title{
Severe Acne Skin Disease: A Fuzzy-Based Method for Diagnosis
}

\author{
Femi Emmanuel Ayo $^{1}$ (D), Roseline Oluwaseun Ogundokun ${ }^{2(\bowtie)}(\mathbb{D}$, \\ Joseph Bamidele Awotunde ${ }^{3}$ (D), Marion Olubunmi Adebiyi ${ }^{2}$, \\ and Abidemi Emmanuel Adeniyi ${ }^{2}$ (D) \\ ${ }^{1}$ Department of Physical and Computer Sciences, McPherson University, \\ Seriki-Sotayo, Abeokuta, Nigeria \\ 2 Department of Computer Science, Landmark University, Omu-Aran, Nigeria \\ ogundokun. roseline@lmu. edu.ng \\ ${ }^{3}$ Department of Computer Science, University of Ilorin, Ilorin, Nigeria
}

\begin{abstract}
Dermis ailments are disorders that hurt or damage the dermis that has an enormous impact on the everyday life of a person. People's tight schedule has significantly impacted their accessibility for repetitive examinations, thereby preventing individuals from consulting a medical practitioner. Network-centered medicinal schemes' popularity is increasingly becoming a model for helping individuals recognize how crucial the level of an ailment is. Acne dermis ailment is one of the extremely well-known dermis sicknesses that troubles the sebaceous glands, thus repetitive diagnosis could assist to avoid blisters. Fuzzy based approach for diagnosing acne skin disease was proposed in this paper. It was suggested that the approach assisted solving the shortcomings of previous expert system methods. Expert machine reasoning is related to literary ambiguity. The proposed system of used fuzzy rules to address inaccuracy in the expert system's analysis. It was proven that the scheme was $82 \%$ accurate, indicating good performance. The Fuzzy expert system built had shown an extreme level of guidance, medical care recommendations and demonstrated the degree of seriousness of acne dermis state in patients.
\end{abstract}

Keywords: Expert system $\cdot$ Diagnosis $\cdot$ Skin disease $\cdot$ Fuzzy logic $\cdot$ Medical system

\section{Introduction}

Skin disease is a skin-exasperating condition which causes signs such as pain, bumping, sweltering and inflammation [1-3]. This can result in complex inferiority and dejection, besides the side effects caused by skin disease. People's schedule has been extremely tight that it affects their ability to perform regular routine check-up with their medical practitioners. An expert system is defined as an artificial intelligence competency that imitates human expert's reasoning ability [4] and has become an alternative to identifying different diseases on the basis of its credibility [5]. Combined with the inability to reveal their health status, the busy schedule for most people has prompted the implementation of a web-based medical system to replace 343 human specialists. 
The widespread adoption of this web-based diagnostic program has required a change from anthropoid meeting to computer meeting. The diagnostic scheme is a device which could classify infections on the basis of the symptoms and their severity [6]. A network-centered diagnostic system is a smart device in rule form [7, 8], with the expertise of some specialist. The software applies these rules to make wise and expert decisions based on some methods for inference [9, 10].

More so, using computer-based devices can go a long way to helping human experts discharge quality healthcare services. Artificial Intelligence (AI) also refers to an intelligent computing, it also refers to designing and implementing machines that act at a human expert level [5]. Compared with other machine learning methods, expert systems have established themselves a niche in AI. In the field of health care $[11,12]$ is intrinsic the most accepted area of application of expert systems for disease detection and control. As the most commonly used tool, they've been given the benefit of problem solving by the pleasant customer interface and description services provided by expert schemes. The system of experts is known for providing explanations, concluding derivatives and emerging from the fire of composite rules [13].

Acne is an illness which irritates the sebaceous glands. Acne happened to be reported as one of the utmost common dermis diseases; at one point or the other, an estimated 80 out of a hundred people have acne. Therefore, the safest way to avoid burns is believed to take early preventive measures $[14,15]$. Several approaches have been used to assess the harshness of acne dermis sickness but most of these methods still lack the ability to accurately categorize the acne frequency [16].

This paper proposed a fuzzy based approach to diagnose acne dermis infection. A fuzzy scheme is an assembly of fuzzy set $[16,17]$ and linguistic variables established on some association purpose described on a certain fluctuating value range. The postulated structure incorporates fuzzy rules, as the administration of improbability in an intrinsically authoritative interest in designing and implementing expert scheme since considerable amount of the datasets available in the comprehension-centered expert scheme is inaccurate, and not entirely reliable [18]. Fuzzy logic is a group of analytical policies for apprehending the interim rates amid the Boolean drastic rates [19, 20]. Fuzzy logic is prepared with regards to accuracy in the representation of reasonable condition, improbability and unsteady structure, ensuing planning and execution of smart schemes [21, 22]. Consequently, the projected scheme of experts entrenched in fuzzy logic would contribute to accuracy, comprehensiveness and accurate endorsements.

The remainder of this manuscript is structured as thus: Sect. 2 describes the related works. Methodology is outlined in Sect. 3 and Sect. 4 outlines the process for implementing and reviewing the proposed method. Section 5 details the conclusion and the future work. 


\section{Literature Review}

\subsection{Acne Skin Disease}

Acne Dermis infection is a long-term dermis condition that happens when hair follicles are obstructed by insensitive dermis cells and dermis oil [23]. It has spots or pustules, oily dermis and likely scratching. Known factors affecting sebum production are a number of Propionibacterium acnes, under the influence of natural circulating Dehydro-Epiandrosterone (DHEA), often activates AV during puberty. Patients seldom, if continuously, grumble concerning decreased sebum creation. However, increased sebum creation, instigating oily dermis which may be an antecedent to acne, is a usual criticism [1]. It is a very popular dermis syndrome that can occur mainly with provocative and non-inflammatory lacerations on the look as well as on the upper arms, the trunk and back. Gender, in particular, has a major and well-known impact, as sebum levels are usually low in infancy, rise in mid-to-late teen years, and remain stable in the seventh and eighth decades before endogenous androgen synthesis fades [1].

Acne can cause emotional distress, and depending on its severity, stain the skin. Acne can cause scarring to the skin but usually does not cause long-term health problems. In the self-corporate image other parts of the body like face play an important role. Even a minor lesion in this portion can be painful to the patient and appear large. This picture can cause mental disorders such as depression and anxiety, low self-esteem and reduced social relationships [1].

Acne mostly appears on the look but is also common on the knees, back and upper arms. Normally, the use of anabolic steroid leads to acne on the shoulders and upper back. The levels of acne severity are four [16]: mild, moderate, moderately extreme and severe (Figs. 1, 2, 3 and 4). Yet even mild acne can be distressing, especially for teenagers who see every pimple as a big cosmetic challenge.

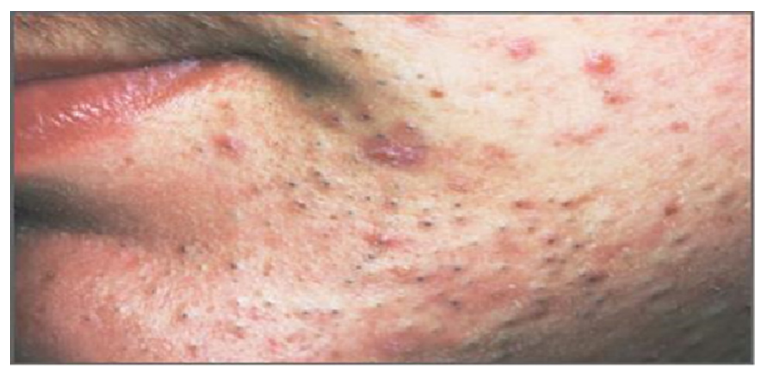

Fig. 1. Minor acne 


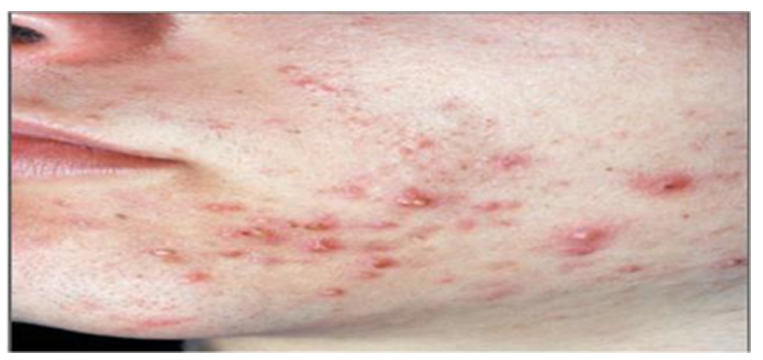

Fig. 2. Average acne

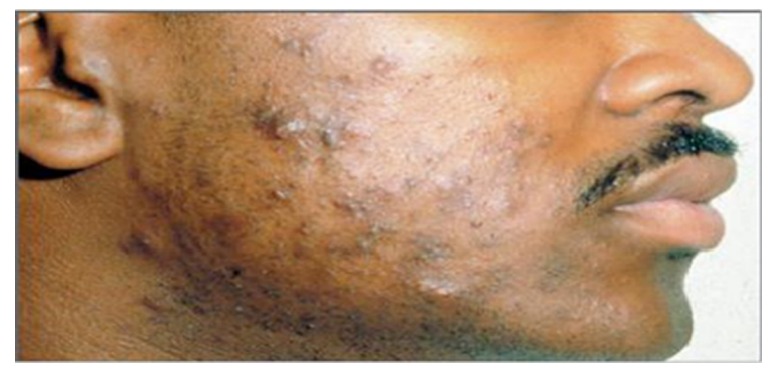

Fig. 3. Averagely harsh acne

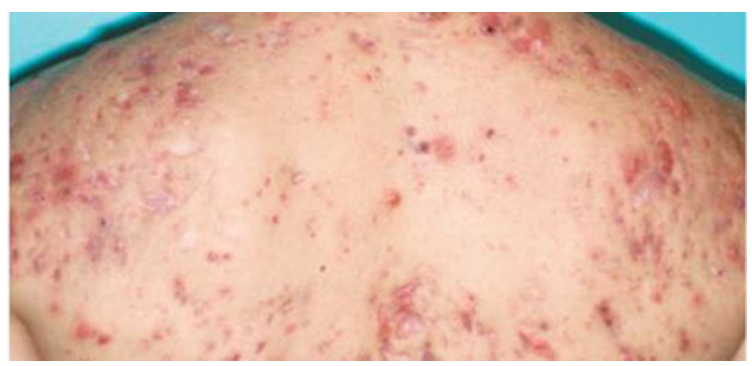

Fig. 4. Harsh acne

Acne severity (Table 1) is mostly considered by the number, size, and dissemination of lacerations (regions with $\mathrm{A}=$ \{blackhead, pimples, pustules, comedones $\}$ (1) wound) [16]. 
Table 1. Severity of acne evaluation

\begin{tabular}{l|l}
\hline Severity & Lesions distribution \\
\hline Mild & $0-10$ \\
\hline Moderate & $10-40$ \\
\hline Moderately severe & $40-100$ \\
\hline Severe & 100 -above \\
\hline
\end{tabular}

\subsection{Fuzzy Logic}

Fuzzy reasoning has proven to be common in control systems. Fuzzy reasoning is designed to apprehend the interim standards amid in the intense Boolean standards $[0$ or 1]. Fuzzy logic is therefore best suited to regulate instability in an unstable system $[16,19,24]$. The University of California at Berkeley first proposed Fuzzy logic [25, 26], also called fuzzy sets, illustrated on his personal beliefs of semantic variables. Fuzzy logic comprises of constituents that includes fuzzy sets, association function, fuzzification, range uncertainty, engine deduction and defuzzification [19, 24].

\subsection{Rule Based Device}

The rule-based systems are employed as an information handling device in computer science [27]. Intellectuals often deploy them in the domain of machine-learning. A perfect instance of a rule-based structure is the knowledge-narrowed, knowledgeable decision-making method. For example, an expert system could improve a human doctor's ability to recommend. Rule-based methods may likewise be intended to run scanners for compiling PC programs, or in natural linguistic procedure [27]. Rulebased programming aims to extract instructions for execution from starting data and rules.

\subsection{Related Works}

[28] developed a system for diagnosing and classifying Acne Lesions in acne patients. The authors introduced numerous image separation techniques for detecting acne lacerations and machine-learning procedures for classifying acne lesions. With good performance accuracy, the designed system had proven to be a good method of detection and classification.

[29] suggested an expert system based on scientific criteria, severity assessment, diagnosis and chronic acne skin disease assessment. The results indicated the capacity of the developed method to diagnose, assess the extent of acne skin disease, and make a distinction between types of acne skin disease.

[30] developed an online expert rule-based structure for the detection and management of skin syndromes. The expert rules were embedded in the warning sign of every kind of dermis syndrome, and introduced employing tree diagram together with deduction exploiting depth-first search approach for forward chaining. The rule-based online expert program showed good results in identification and proved timely for diagnosing thirteen plus skin diseases. 
[31] Fuzzy inferencing system designed and implemented to diagnose skin disease in infants. The writers dealt with selected infectious disorders, such as measles, German measles and chicken pox. The reason selected diseases were chosen was due to their infection resemblances and symptoms such as rash and fever. The authors based their Fuzzy inference system design on rules developed from the knowledge and literature of experts. The system's implementation had shown the ability to diagnose skin diseases correctly in infants.

[32] presented a mobile medical aid system to diagnose skin diseases by using casebased reasoning to deduce a new rule in the database and image processing technique to determine symptoms based on a newly captured picture of an infected skin. The work was designed to provide patients with infected skin with pre-examination assistance and increase awareness of skin diseases and their effects. This method was found to be effective in the early detection and treatment of problems with skin diseases.

[33] developed a novel spontaneous diagnostic approach for facial acne vulgaris, rooted in convolutionary neural networks for extraction and classification of features. The aim was to resolve the shortcomings of previous approaches of mostly expert systems which lack the ability to distinguish enough types of acne vulgaris due to the inaccuracy nature of the design of the expert system. The results showed that the neural network is operational to eliminate features from the images of facial acne vulgaris and to strengthen classifiers for proper classification.

\section{Materials and Method}

\subsection{Datasets}

For this work, the data set used was originally generated in accordance with expert guidelines and literature regulations.

\subsection{Methodology}

The Fuzzy logic was used in this study to develop a specialist scheme based on some identified symptoms to diagnose the gravity of acne dermis infection. Expert methodological detections and thoughts were filled with improbability from the literature, which is why the authors used fuzzy logic to propose solutions to these uncertainties.

\subsection{Proposed Architecture Model}

Figure 5 illustrates the projected system architecture for the detection of severity of acne dermis infection. The architectural model generates acne-related symptoms from the sufferer by means of the user interface and established on the sufferer feedbacks, the system converts the feedbacks to fuzzy valves and then employs its inference device established on the rules within the expert system to decide on the gravity of the acne dermis infection. 


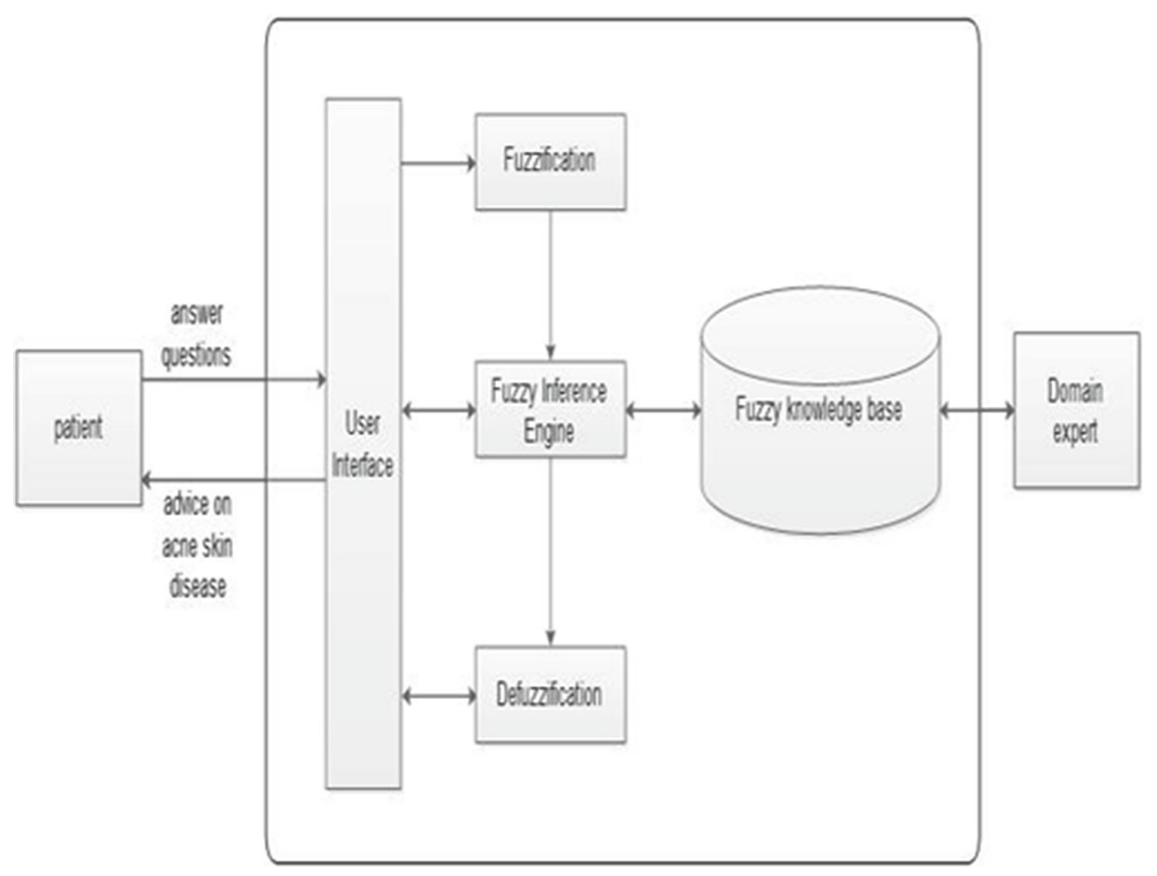

Fig. 5. System architecture

Table 2 reflects the procedures in the postulated fuzzy knowledge centred scheme, utilizing their corresponding etymological variables (small, medium, big, and very large). Within the knowledge base of the program, expert expertise in the area were used to shape the rules.

Table 2. Sample rule based

\begin{tabular}{l|l|l|l|l|l}
\hline \#no & Blackhead & Pimples & Pustules & Comedones & Conclude \\
\hline 1 & Very large & Very large & Very large & Very large & Severe \\
\hline 2 & Medium & Large & Large & Large & Moderately severe \\
\hline 3 & Medium & Medium & Medium & Medium & Moderate \\
\hline 4 & Small & Small & Small & Small & Mild \\
\hline
\end{tabular}


As shown in Fig. 6, the knowledge centred for the projected fuzzy network of experts (Table stores the details, laws and guidelines on the symptoms).

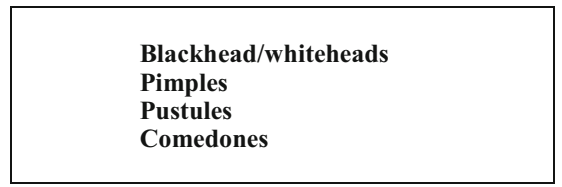

Fig. 6. Acne symptoms

Accordingly, a modest clarification for rule 2 of Table 2 was: IF blackhead is medium together with pimples being large as well as pustules being large, THEN acne is moderate (comedones therefore are large). For the detection of intensity of the acne dermis infection, the illustrative rule in Table 2 above were a depiction of the knowledge centred system which comprises of rules in the projected fuzzy specialist scheme. The fuzzy varying rate amid the interval $[0,1]$ for the language variables of the postulated fuzzy sets is shown in Table 3. Likewise, Table 3 also indicates the severity spectrum of the lesions being distributed.

Table 3. Fuzzy range value

\begin{tabular}{l|l|l|l}
\hline Linguistic variables & Linguistic range & Lesions & Lesions range \\
\hline Small & $0.1 \leq \mathrm{x}<0.3$ & Mild & $0-10$ \\
\hline Medium & $0.3 \leq \mathrm{x}<0.6$ & Moderate & $10-40$ \\
\hline Large & $0.6 \leq \mathrm{x}<0.8$ & Moderately severe & $40-100$ \\
\hline Very large & $0.8 \leq \mathrm{x}<0.1$ & Severe & 100 -above \\
\hline
\end{tabular}

\section{Result and Discussion}

This study employed java programming language for the implementation of the proposed system. and Mysql database for storage. A login feature was introduced to the fuzzy expert structure developed for detecting acne dermis infection and this was illustrated in the Fig. 7. Java programming language was employed for the front end of the proposed scheme whereas Mysql was employed for the back end and this involves the user login feature introduced. 


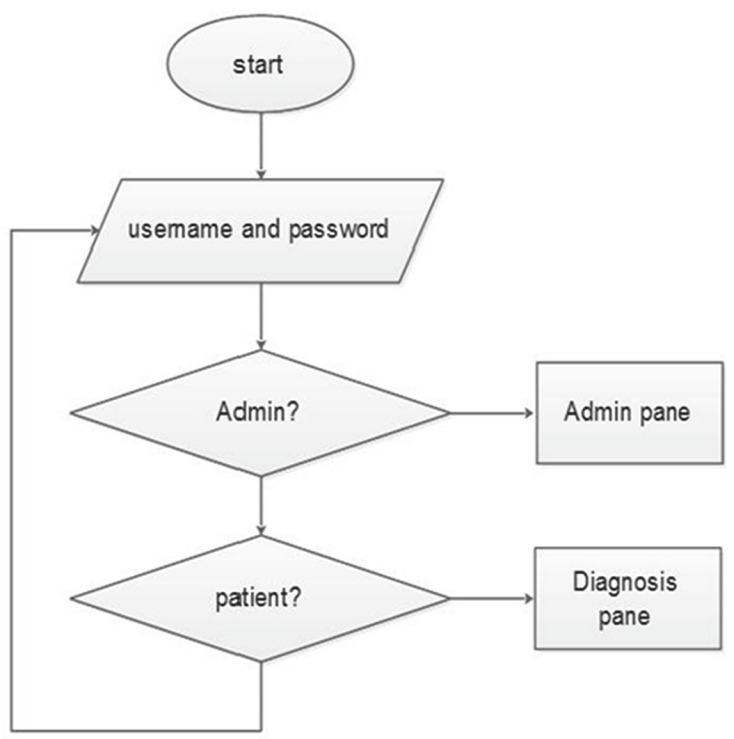

Fig. 7. Login flowchart

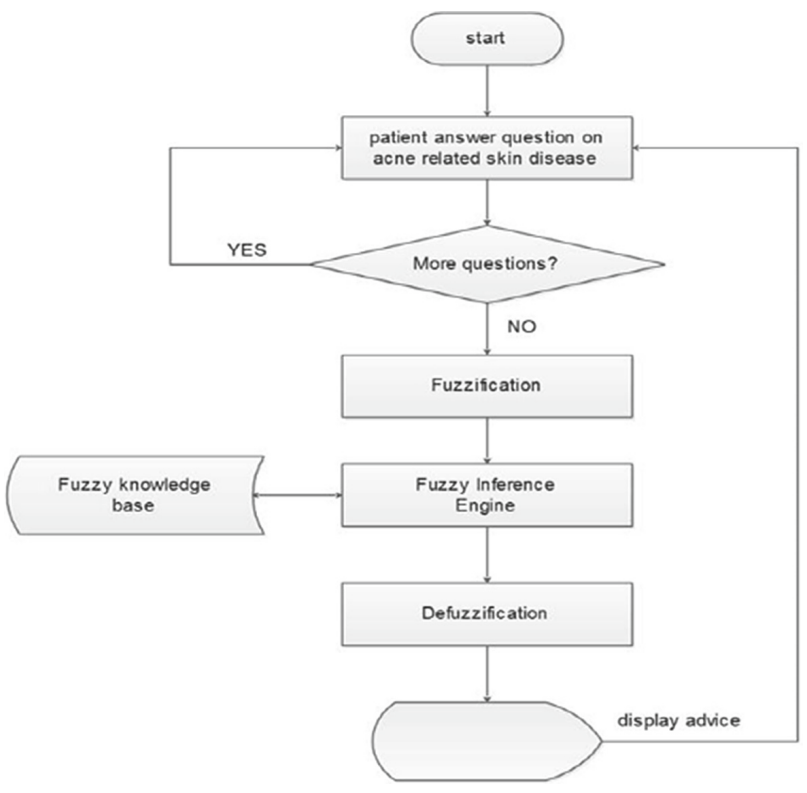

Fig. 8. System flowchart 
Figure 8 shows the flowchart of the proposed system.

The Fuzzy expert program introduced assisted in establishing if an individual has acne dermis infection and categorize the acne intensity as mild, moderate or extreme and this was established on the amount and nature of the indications. The system was implemented with Intel Dual core $2.20 \mathrm{GHz}$ processor on a PC workstation. An interface for the machine login pane is shown in Fig. 9. The device users (sufferers) were necessitated to validate their authorization into the system with their username and password information via the LOGIN button. A first timer can as well sign-up for future authentication in the system via the REGISTER button.

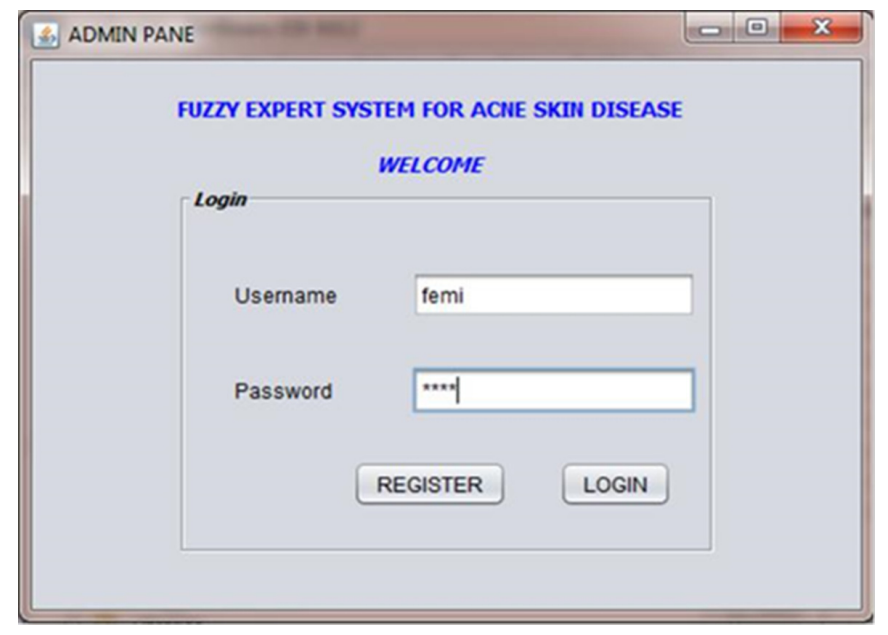

Fig. 9. Login interface

Figure 10 shows the Fuzzy Expert System detection and outcome pane. After a successful login the user was taken to this pane. The program elicits user-related acneassociated sickness problems. An individual is required to select answers and a variety of ambiguous linguistic alternatives that the program can employ to interpret, and to make suggestion in form of management. The outcome was revealed in the system's RESULT section, once a DIAGNOSE button was clicked. 


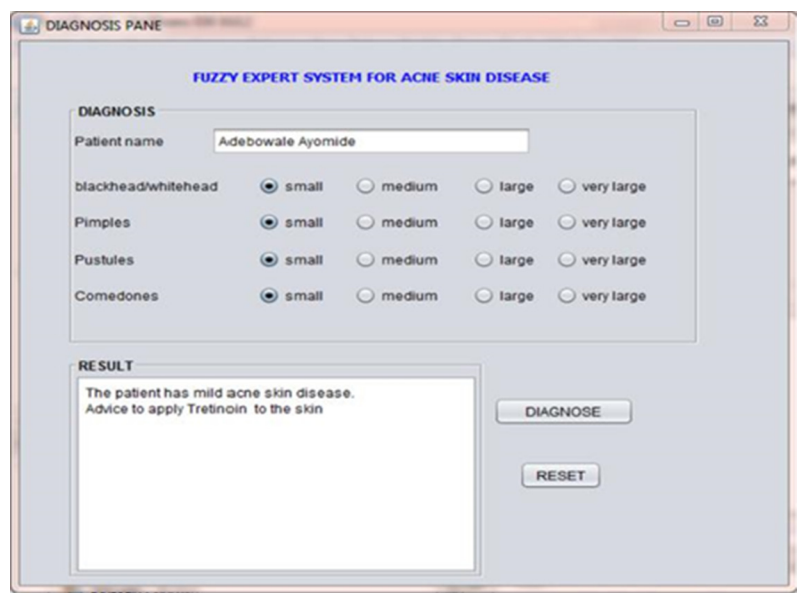

Fig. 10. Detection and outcome pane

The chosen permutation in Fig. 10 demonstrated the patient's diagnosis of the fuzzy expert method, with minor acne dermis infection and Tretinoin medication to be administered to the dermis infected. The user login database is shown in Fig. 11.

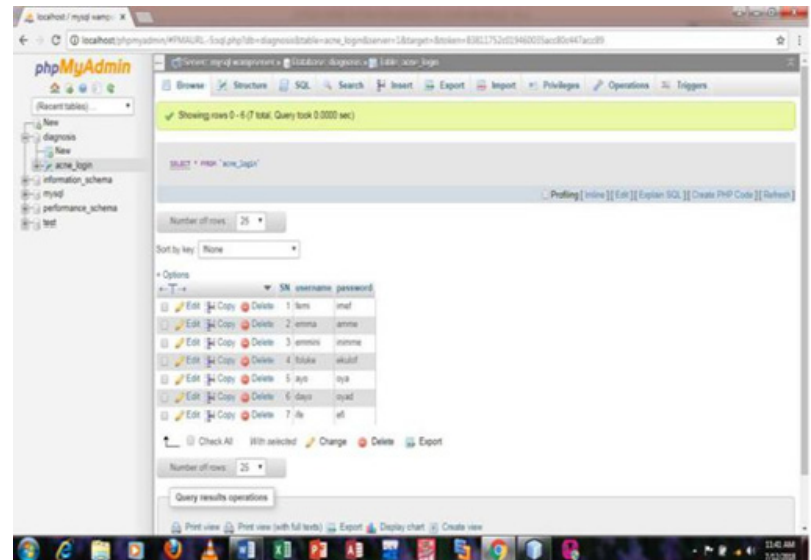

Fig. 11. Login database 


\section{Performance Evaluation}

For the severity of the acne dermis syndrome, this paper adopted the following modelling process to validate the analysis precision of the projected fuzzy expert scheme. A confusion matrix which contained information that is needed for a classification system assessment. Table 4 presents a typical confusion matrix metrics. The system's prediction of acne severity was evaluated using those metrics. Therefore, severity had to be classified by lesion distribution. The information in Table 5 indicated the classification results, as the program predicted. For machine results, the uncertainty matrix is as shown in Table 6.

Table 4. Confusion matrix metrics

\begin{tabular}{l|l|l|l}
\hline \multicolumn{5}{l}{ True class } & Positive & Negative & Total \\
\hline \multirow{2}{*}{$\begin{array}{l}\text { Predictive } \\
\text { class }\end{array}$} & $\begin{array}{l}\text { True Positive Count } \\
\text { (TP) or Severe }\end{array}$ & $\begin{array}{l}\text { False Positive Count } \\
(\text { FP) or Moderately Severe }\end{array}$ & TP + FP \\
\cline { 2 - 4 } & $\begin{array}{l}\text { False Negative Count } \\
\text { (FN) or Moderate }\end{array}$ & $\begin{array}{l}\text { True Negative Count } \\
(\text { TN }) \text { or Mild }\end{array}$ & FN + TN \\
\hline Total & TP + FN & FP + TN & TP + FN + FP + FN \\
\hline
\end{tabular}

Table 5. The results of system grading for 100 Cases

\begin{tabular}{l|l|c}
\hline Lesions & Classification & Count \\
\hline $0-10$ & True negative or mild & 36 \\
\hline $10-40$ & False negative or moderate & 16 \\
\hline $40-100$ & False positive or moderately severe & 2 \\
\hline $100-$ above & True positive or severe & 46 \\
\hline
\end{tabular}

Table 6. Confusion matrix for 100 cases

\begin{tabular}{l|l|l|l}
\hline & \multicolumn{2}{|l|}{$\begin{array}{l}\text { Observation } \\
\text { class }\end{array}$} & Total \\
\hline Positive & TP & FP & 48 \\
& 46 & 2 & \\
\hline Negative & FN & TN & 52 \\
& 16 & 36 & \\
\hline Total & 62 & 38 & 100 \\
\hline
\end{tabular}


With the confusion matrix in Table 6 , the probability of true positive rate, true negative rate and accuracy rate were computed as follows:

Probability of true positive rate

$$
\mathrm{TP} /(\mathrm{TP}+\mathrm{FN})=46 /(46+16)=0.7419
$$

Probability of true negative rate

$$
\mathrm{TN} /(\mathrm{TN}+\mathrm{FP})=36 /(36+2)=0.9474
$$

Probability of Accuracy rate

$$
(\mathrm{TP}+\mathrm{TN}) /(\mathrm{TP}+\mathrm{TN}+\mathrm{FP}+\mathrm{FN})=46+36 /(46+36+2+16)=0.82
$$

\section{Conclusion}

The purpose of this paper was to improve people's check-up habits by designing a friendly and interactive fuzzy skin disease diagnostic expert system. Users can use the service provided by the designed fuzzy expert system to diagnose and get an idea of the severity of the disease that affects them. Using the evaluation results of Table 6, which includes the confusion matrix for 100 system classification cases, we can then deduce that the designed system showed a true positive rate, true negative rate, and accuracy rate of $0.7419,0.9474$, and 0.82 respectively. The conclusion taken from this result is that the device obtained an accuracy rate of $82 \%$ after the confusion matrix assessment process. This indicated a substantial good rating for the fuzzy expert method, and an indicator of its usefulness to help the mission of predicting the extent of acne skin disease, as well as providing advice on care.

\section{Future Work}

In the future, the authors will implement the use of adaptive neuro-fuzzy inference method for the same mission and then will compare the results.

\section{References}

1. Ak, M.: A comprehensive review of acne vulgaris. J. Clin. Pharm. 1(1), 17-45 (2019)

2. Pereira, M.P., Kremer, A.E., Mettang, T., Ständer, S.: Chronic pruritus in the absence of skin disease: pathophysiology, diagnosis and treatment. Am. J. Clin. Dermatol. 1-12. Clerk Maxwell, J.: A Treatise on Electricity and Magnetism, 3rd edn., vol. 2. Oxford: Clarendon, 1892, 2016, pp. 68-73

3. Adeyinka, A.A., Adebiyi, M.O., Akande, N.O., Ogundokun, R.O., Kayode, A.A., Oladele, T.O.: A deep convolutional encoder-decoder architecture for retinal blood vessels segmentation. In: Misra, S., et al. (eds.) ICCSA 2019. LNCS, vol. 11623, pp. 180-189. Springer, Cham (2019). https://doi.org/10.1007/978-3-030-24308-1_15 
4. Habif, T.P., Chapman, M.S., Campbell Jr., J.L., Dinulos, J.G., Zug, K.A.: Skin disease: diagnosis and treatment. Elsevier Health Sciences, pp. 201-214 (2011)

5. Yadav, G., Pandey, G.N.: Development of intelligent decision and prediction system using cyber-enabled. NESS Technology for oil availability and yield prediction. RIET-IJSET: Int. J. Sci. Eng. Technol. 2(1), 41-52 (2015)

6. Amarathunga, A.A.L.C., Ellawala, E.P.W.C., Abeysekara, G.N., Amalraj, C.R.J.: Expert system for diagnosis of skin diseases. Int. J. Sci. Technol. Res. 4(1), 456-472 (2015)

7. Lingiardi, V., McWilliams, N., Bornstein, R.F., Gazzillo, F., Gordon, R.M.: The psychodynamic diagnostic manual version 2 (PDM-2): assessing patients for improved clinical practice and research. Psychoanalytic Psychol. 32(1), 94 (2015)

8. Moses, D.: A survey of data mining algorithms used in cardiovascular disease diagnosis from multi-lead ECG data. Kuwait J. Sci. 42(2) (2015)

9. Adegun, A.A., Akande, N.O., Ogundokun, R.O., Asani, E.O.: Image segmentation and classification of large scale satellite imagery for land use: a review of the state of the arts. Int. J. Civil Eng.Technol. 9(11) (2018)

10. Ajala, F.A., Akande, N.O., Adeyemo, I.A., Ogundokun, R.O.: Smallest univalue segment assimilating nucleus approach to brain MRI image segmentation using fuzzy C-means and fuzzy K-means algorithms. Int. J. Comput. Technol. 16(7), 7065-7076 (2017)

11. Ayo, F.E., Awotunde, J.B., Ogundokun, R.O., Folorunso, S.O., Adekunle, A.O.: A decision support system for multi-target disease diagnosis: a bioinformatics approach. Heliyon 6(3), e03657 (2020)

12. McPherson, R.A., Pincus, M.R.: Henry's clinical diagnosis and management by laboratory methods. Elsevier Health Sciences (2016)

13. Jimoh, R.G., Awotunde, J.B., Babatunde, A.O., Ameen, A.O, James, T.R., Fatai, O.W.: Simulation of medical diagnosis system for malaria using fuzzy logic. Int. J. Inf. Process. Commun. (IJIPC) 2(1) (2014). Published by Faculty of Communication and Information Sciences, University of Ilorin, Ilorin

14. Horvitz, E.J., Breese, J.S., Henrion, M.: Decision theory in expert systems and artificial intelligence. Int. J. Approximate Reasoning 2(3), 247-302 (1988)

15. Constantinou, A.C., Fenton, N., Marsh, W., Radlinski, L.: From complex questionnaire and interviewing data to intelligent Bayesian network models for medical decision support. Artif. Intell. Med. 67, 75-93 (2016)

16. Ayo, F.E., et al.: A fuzzy based method for diagnosis of acne skin disease severity. imanager's. J. Pattern Recogn. 5(2), 10 (2018)

17. Adebayo, O., Asani, E.O., Ogundokun, R.O., Ananti, E.C., Adegun, A.: A neuro-fuzzy based system for the classification of cells as cancerous of non-cancerous. Int. J. Med. Res. Health Sci. 7(5), 155-166 (2018)

18. Thompson, T., Sowunmi, O., Misra, S., Fernandez-Sanz, L., Crawford, B., Soto, R.: An expert system for the diagnosis of sexually transmitted diseases-ESSTD. J. Intell. Fuzzy Syst. 33(4), 2007-2017 (2017)

19. Jimoh, R.G., Afolayan, A.A., Awotunde, J.B., Matiluko, E.O.: Fuzzy logic based expert system in the diagnosis of ebola virus. Ilorin J. Comput. Sci. Inf. Technol. 2(1), 73-94 (2017). Published by Computer Science Department, University of Ilorin, Ilorin, Nigeria

20. Azeez, N.A., Towolawi, T., Van der Vyver, C., Misra, S., Adewumi, A., Damaševičius, R., Ahuja, R.: A fuzzy expert system for diagnosing and analyzing human diseases. In: Abraham, A., Gandhi, N., Pant, M. (eds.) IBICA 2018. AISC, vol. 939, pp. 474-484. Springer, Cham (2019). https://doi.org/10.1007/978-3-030-16681-6_47 
21. Lawanya Shri, M., Ganga Devi, E., Balusamy, B., Kadry, S., Misra, S., Odusami, M.: A fuzzy based hybrid firefly optimization technique for load balancing in cloud datacenters. In: Abraham, A., Gandhi, N., Pant, M. (eds.) IBICA 2018. AISC, vol. 939, pp. 463-473. Springer, Cham (2019). https://doi.org/10.1007/978-3-030-16681-6_46

22. Alhassan, J.K., Misra, S., Umar, A., Maskeliūnas, R., Damaševičius, R., Adewumi, A.: A fuzzy classifier-based penetration testing for web applications. In: Rocha, Á., Guarda, T. (eds.) ICITS 2018. AISC, vol. 721, pp. 95-104. Springer, Cham (2018). https://doi.org/10. 1007/978-3-319-73450-7_10

23. Adegun, A.A., Ogundokun, R.O., Adebiyi, M.O., Asani, E.O.: CAD-based machine learning project for reducing human-factor-related errors in medical image analysis. In: Misra, S., Adewumi, A. (eds.) Handbook of Research on the Role of Human Factors in IT Project Management, pp. 164-172. IGI Global, Hershey (2020). https://doi.org/10.4018/978-17998-1279-1.ch011

24. Awotunde, J.B., Matiluko, O.E., Fatai, O.W.: Medical diagnosis system using fuzzy logic. Afr. J. Comput. ICT 7(2), 99-106 (2014). Published by IEEE Computer Society, Nigeria Section

25. Jang, J.S.: Neuro-Fuzzy Modeling: Architectures, Analyses, and Applications. University of California, Berkeley (1992)

26. Bo, Y. (ed.): Fuzzy Sets, Fuzzy Logic, and Fuzzy Systems: Selected Papers by Lotfi A Zadeh, vol. 6. World Scientific (1996)

27. Hachaj, T., Ogiela, M.R.: Rule-based approach to recognizing human body poses and gestures in real time. Multimedia Syst. 20(1), 81-99 (2013). https://doi.org/10.1007/s00530013-0332-2

28. Alamdari, N., Tavakolian, K., Alhashim, M., Fazel-Rezai, R.: Detection and classification of acne lesions in acne patients: a mobile application. In: 2016 IEEE International Conference on Electro Information Technology (EIT), pp. 0739-0743. IEEE, May 2016

29. Zouboulis, C.C., Del Marmol, V., Mrowietz, U., Prens, E.P., Tzellos, T., Jemec, G.B.: Hidradenitis suppurativa/acne inversa: criteria for diagnosis, severity assessment, classification and disease evaluation. Dermatology 231(2), 184-190 (2015)

30. Asghar, M.Z., Asghar, M.J., Saqib, S.M., Ahmad, B., Ahmad, S., Ahmad, H.: Diagnosis of skin diseases using online expert system. Int. J. Comput. Sci. Inf. Secur. 9(6), 323 (2011)

31. Putra, A.A., Munir, R.: Implementation of fuzzy inference system in children skin disease diagnosis application. In: 2015 International Conference on Electrical Engineering and Informatics (ICEEI), pp. 365-370. IEEE, August 2015

32. Aruta, C.L., Calaguas, C.R., Gameng, J.K., Prudentino, M.V., Anthony, A., Lubaton, C.J.: Mobile-based medical assistance for diagnosing different types of skin diseases using casebased reasoning with image processing. IJ CI 3(3), 115-118 (2015)

33. Shen, X., Zhang, J., Yan, C., Zhou, H.: An automatic diagnosis method of facial acne vulgaris based on convolutional neural network. Sci. Rep. 8(1), 1-10 (2018) 